\title{
Evaluation of the American Physical Therapy Association's Guidelines for Training Culturally Competent Physical Therapists
}

\author{
James McKivigan \\ School of Physical Therapy, Touro University, Henderson, USA
}

Email address:

james.mckivigan@tun.touro.edu

\section{To cite this article:}

James McKivigan. Evaluation of the American Physical Therapy Association's Guidelines for Training Culturally Competent Physical Therapists. Higher Education Research. Vol. 5, No. 4, 2020, pp. 154-161. doi: 10.11648/j.her.20200504.16

Received: June 21, 2020; Accepted: July 20, 2020; Published: July 23, 2020

\begin{abstract}
Background: Training healthcare practitioners on cultural competence and increasing the cultural diversity of healthcare professionals, may help to alleviate barriers and improve patient outcomes. The American Physical Therapy Association (APTA) has implemented goals and guidelines for the training of culturally competent physical therapists. The goal of this study was to evaluate whether an association exists between physical therapy directors' self-reports of the implementation of the APTA goals and guidelines and the cultural competence of those directors, and also to examine whether there are racial/ethnic differences in the implementation of these goals and guidelines and cultural competency among the directors. Methods: The study design was a cross sectional. The survey was sent to 225 physical therapy directors who were required to provide information about the implementation of the APTA goals and guidelines, cultural competency, and demographic characteristics. Linear regression was used to test the association between the implementation of APTA goals and guidelines and the cultural competence of the physical therapy directors. Independent sample t-tests were used to examine whether there were racial/ethnic differences in the implementation of these goals and guidelines and the cultural competence of the directors. Results: A total of 47 physical therapy directors responded to the survey. There was no significant relationship between the implementation of APTA goals and guidelines and the cultural competence of the physical therapy directors or significant racial/ethnic differences in the implementation of the goals and guidelines and cultural competence among the directors. Conclusions: Recommendations for practicing cultural competency in the field of physical therapy will need to be supported by further research into other populations. Other frameworks for understanding cultural competence among physical therapists should be explored.
\end{abstract}

Keywords: Physical Therapy, Cultural Competence, Guidelines, Program Directors

\section{Introduction}

The U.S. population has increasingly grown more racially and ethnically diverse [1-3]. Minorities account for almost one-third of the U.S. population, and are projected to rise to $54 \%$ by 2050 . The U.S. Census Bureau projects that by the middle of the 21 st century, existing minority groups would account for more than $50 \%$ of the population [4]. The diversity of the U.S. population also permeates the healthcare environment [5]. Patients have a variety of religious, socioeconomic, and cultural backgrounds, as well as values, beliefs, and behaviors [5]. This diversity can be a problem for healthcare providers as the culture and values of patients differ from those of the healthcare providers. This is often the case because the healthcare sector does not reflect the same diversity as the one in the United States [6].

Cultural diversity affects the types of diseases and conditions people develop due to genetics, lifestyle, eating habits, location, and other factors [7-11]. Although diseases can affect a wide range of people, the prevalence of these diseases can be more pronounced in specific racial and ethnic groups [10]. As the number of ethnic minority individuals increases, whether from indigenous or immigrant groups, and meet the status of senior citizen, health and medical care professionals need to take into account the cultural background of these individuals because their cultural 
background could determine their expectations and needs, and may factor into the necessary and appropriate courses of treatment. Healthcare providers can help to improve patient care by understanding the relationship between a patient's culture and beliefs and the likelihood of having certain diseases and conditions. Therefore, cultural awareness becomes much critical in establishing a relationship to provide the competent care needed by culturally diverse patients.

A person's cultural background affects how he or she views the definitions of health and disease, and seeking treatment for pain and discomfort is often centered on cultural norms. This situation may pose a problem if the healthcare provider is unable or unwilling to understand the values and beliefs of patients. Providers who are unaware of personal assumptions and biases are often unable to tailor patient information according to the patient's level of understanding or lack effective communication skills [12]. Although one of the objectives of the Affordable Care Act (ACA) was to eliminate disparities in healthcare by promoting equitable access to healthcare, particularly in terms of patient care, studies have shown that there are still disparities in healthcare access, quality, and outcomes [13].

Cultural competence is defined as "the process by which the healthcare professional continually strives to achieve the ability and availability to function effectively within the cultural context of the client, family, individual, and community [14]." Although this concept was initially applied to nurses [15], it has been widely accepted in the medical field and has become increasingly vital amidst the changing demographics of the United States [16-18]. Researchers have emphasized the need for healthcare professionals to develop cultural competence to enhance the delivery of culturally competent healthcare $[19,20]$. This concept now appears in the training of medical professionals $[17,21]$ and the ACA referred to the incorporation of cultural competence in medical training $[4,13]$.

Healthcare professionals face a range of obstacles in developing cultural competence that may be harmful to patient safety, including lack of information about the culture, values, and beliefs of others; negative emotional response, such as anxiety when encountering a member of a different cultural group; prejudice against another's culture and beliefs; and language barriers [22]. The development of cultural competence is in stages, beginning with cultural destructiveness and ending with cultural proficiency. In cultural proficiency stage, a healthcare professional understands the need to conduct research relating to cultural competence in order to disseminate results and to develop new approaches to providing culturally competent care [5, 23].

The Commission on Accreditation of Physical Therapy Education has cultural competency training requirements [18]. The APTA aims to educate physical therapists to be culturally competent to enhance the principals of core professional values, as well as to address the ever-increasing factors of diversity and globalization in the healthcare field
$[24,25]$. The APTA states that for a system to become culturally competent, it must value diversity, have the capacity for cultural self-assessment, be conscious of the dynamics inherent when cultures interact, institutionalize cultural knowledge and develop adaptations for service delivery that reflect an understanding of diversity between and within cultures [24]. With only "blueprints" at their disposal, educational institutions are responsible for developing their definitive curricula for teaching cultural competence in compliance with the APTA regulations. There are many models for implementing cultural competence education. However, there appears to be no medical and healthcare standard accepted as the best practice. Although most models addressed the essential components of cultural competence and were useful in the absence of other programs [18], there was no evidence supporting the efficacy of these initiatives in improving healthcare professionals' cultural competence.

With the increasing importance of cultural competence in the medical field and the introduction of the APTA goals and guidelines for the training of culturally competent physical therapists, there is a need to understand the role that these goals and guidelines might play in increasing cultural competence of physical therapy directors and the factors that influence the implementation of these goals and guidelines and cultural competence. Therefore, this study seeks to (a) evaluate whether an association exists between the selfreported implementation of APTA goals and guidelines and the cultural competence of physical therapy directors and (b) examine whether there are racial/ethnic differences in the implementation of these goals and guidelines and the cultural competence of program directors.

\section{Methods}

\subsection{Sample}

APTA is an individual membership professional organization representing more than 95,000 members of physical therapists, physical therapist assistants, and students. An e-mail survey was sent to 225 directors of physical therapy programs whose email addresses were available on the APTA website. A sample size calculation was used to determine the minimum sample size needed to obtain a representative sample from this population, which indicated that a sample of 140 participants drawn from the sampling frame of 225 would provide a $95 \%$ confidence level with a $5 \%$ margin of error.

\subsection{Instrumentation}

The researcher-developed survey included 10 items, based on the 10 major requirements for training in cultural competence set out in the goals and guidelines of the APTA. Items related to the expectations for cultural competence are statements beginning with "I" to indicate an action. Participants responded using a Likert scale ranging from 1 (Strongly Disagree) to 5 (Agree). The questionnaire 
contained 10 statements with a potential total score of 10 to 50 for each respondent. A score of 10 represents the lowest level of meeting cultural competence training requirements, and a score of 50 represents the highest level of meeting cultural competence training requirements. An additional 20 items on the questionnaire were based on Portalla and Chen's [26] 20-item Intercultural Effectiveness Scale (IES), measuring six dimensions of cultural competence: behavioral flexibility, identity maintenance, interaction management, interaction respect, interaction relaxation, and massage skills. These items were measured with a five-point Likert scale, ranging from 1 (Strongly Disagree) to 5 (Agree). Nine of the 20 negatively-worded survey items were reverse coded. The scores were averaged for the constituent items, generating a scale from 1 to 5 , where higher scores indicate greater levels of cultural competence. Previously, research has shown a high degree of reliability of this instrument, with one paper finding that it has a Cronbach alpha reliability coefficient of 0.85 , indicating that there is a high degree of consistency between the individual items which comprise the scale [26]. Individuals who scored high on the IES were behaviorally adaptive, able to distinguish between appropriate behaviors, and able to adapt to different situations [26]. Lastly, respondents' ethnicity was measured using an additional item on the questionnaire that required each participant to identify with a particular ethnic/racial group: White/Caucasian, African American, Hispanic, Asian, Mixed Race, or other. Responses of White/Caucasian will be considered as White and responses of African American, Hispanic, Asian, Mixed Race, or others will be regarded as non-White. This action was necessary because independent samples of $t$-tests can only be conducted if at least two participants in each group are compared.

In order to ensure the reliability and validity of the questionnaire, a field test was conducted prior to interviews to determine any limitations on the questionnaire, the wording of the items and the appropriateness; to ensure that participants understood the items of the questionnaire as intended [27, 28]. These field test interviews were conducted with two directors of physical therapy programs who were not part of the primary study. After completing the questionnaire, each field participant was asked to reassess any item that needed clarification. Results from the field test were recorded, and some adjustments were made to the questionnaire. For distribution to the sampling frame, the survey was converted into a Google form.

\subsection{Data Collection}

The researcher sent messages containing the survey to the 225 APTA directors in the sampling frame via e-mail. Informed consent forms were collected before any data was obtained from the participants. Participants electronically consented to participate in the survey.

\subsection{Analysis}

The researcher imported the data from the Google forms spreadsheet into SPSS v. 20.0. Descriptive statistics, including, frequency distribution, minimums, maximums, means, medians, and standard deviation were computed to summarize the total scores for the ten items in the Requirements for Education in Cultural Competence in Physical Therapy and the six dimensions of the 20-item Intercultural Effectiveness Scale. Analysis of Variance (ANOVA) tests were performed to check for differences in mean scores on these two measures according to any of the demographic characteristics. These tests were conducted to identify the appropriateness of including the demographic characteristics as covariates in the analyses.

To address whether there is a relationship between the implementation of APTA goals and guidelines and the cultural competence of the physical therapy directors, a simple linear regression analysis was performed in which the cultural competence of the program director was included in the analysis as the independent variable and the implementation of APTA goals and guidelines was included as the dependent variable. T-tests were performed to test for racial/ethnic differences in mean score for the implementation of APTA goals and guidelines and cultural competence score.

A post hoc power analysis was conducted to determine the power of the study findings based on the actual sample size and effect size of the results using WebPower [29], a webbased power analysis tool that allows for calculations of power based on inputting the sample size, effect size, probability of type 2 error $(p<.05)$, and the observed relationship between the variables.

\section{Results}

Of the 225 physical therapy directors to whom the survey was sent, 55 clicked on the survey link and agreed to participate. Of these 55, 2 did not answer any survey questions, and 6 responded only to the questions on demographic characteristics without completing the questions on the implementation of APTA goals and guidelines or cultural competence. This situation resulted in a total sample of 47 participants who completed all aspects of the survey.

Table 1. Participant demographic characteristics.

\begin{tabular}{lll}
\hline Characteristic & $\boldsymbol{n}$ & $\mathbf{\%}$ \\
\hline Age & 5 & \\
35-44 years & 22 & 9.4 \\
45-54 years & 23 & 41.5 \\
55-64 years & 3 & 43.4 \\
65-74 years & & 5.7 \\
Race/ethnicity & 46 & \\
White/Caucasian & 2 & 86.8 \\
Black/African American & 1 & 3.8 \\
Hispanic & 1 & 1.9 \\
Asian/Pacific Islander & 3 & 1.9 \\
Other & & 5.7 \\
Education & 0 & \\
Less than high school & 0 & 0.0 \\
Bachelor's degree & 54 & 0.0 \\
Graduate degree & & 100.0 \\
\hline
\end{tabular}




\begin{tabular}{lll}
\hline Characteristic & $\boldsymbol{n}$ & $\mathbf{\%}$ \\
\hline Employment status & & \\
Employed full time & 54 & 100.0 \\
Employed part time & 0 & 0.0 \\
Unemployed & 0 & 0.0 \\
Retired & 0 & 0.0 \\
Marital status & & \\
Married & 46 & 88.5 \\
Single/divorced & 6 & 11.5 \\
Income & & \\
Less than $\$ 100,000$ & 1 & 2.0 \\
\$100,000-\$119,999 & 7 & 14.3 \\
\$120,000-\$139,999 & 9 & 18.4 \\
\$140,000-\$159,999 & 7 & 14.3 \\
More than $\$ 160,000$ & 25 & 51.0 \\
\hline
\end{tabular}

As shown in Table 1, participants identified predominantly as White/Caucasian, all had a graduate degree, and were all employed full time. The majority of participants were married, with a small group either single or divorced. Participants' income skewed towards the higher end of the scale, with just over half of the participants making more than $\$ 160,000$ a year.

Descriptive statistics for the implementation of APTA guidelines of the participants' cultural competency are presented in Table 2. The scores for participants implementation of the APTA guidelines ranged from 34 to 48 . The mean and the median were 42 and 43, respectively. For the level of cultural competency, participants mean scores ranged from 3.15 to 5.00 . The overall mean and median scores were 4.10 and 4.00 , respectively.

Table 2. Descriptive Statistics for the Continuous Study Measures.

\begin{tabular}{|c|c|c|c|c|c|c|}
\hline Construct & $n$ & Range & $M(S D)$ & Median & Skewness & Kurtosis \\
\hline Implementation of APTA guidelines & 47 & $34.00-48.00$ & $42.26(4.07)$ & 43.00 & -0.46 & -0.62 \\
\hline Cultural competence & 47 & $3.15-5.00$ & $4.10(0.50)$ & 4.00 & 0.33 & -0.70 \\
\hline
\end{tabular}

Note. $M=$ mean; $S D=$ standard deviation.

To ensure that t-tests and linear regression could be performed, the statistical assumptions included in these models have been tested. It was concluded that there were no significant outliers that could have had an effect on these tests, that the variables were normally distributed, and that covariance between the variables was not a significant issue.

The results of the ANOVA test conducted to determine whether there were differences in mean scores on the implementation of APTA guidelines and cultural competence according to any of the demographic characteristics indicated that there was no difference by age in adherence to the APTA guidelines $(F=0.35, p=0.79)$ or cultural competence $(F=0.85$, $p=0.48)$. Mean scores did not differ by income for adherence to the APTA guidelines $(F=0.36, p=0.79)$ or for cultural competence $(F=1.72, p=0.18)$. Mean scores on adherence to the APTA guidelines differed at the trend level between married participants and the single/divorced $(F=3.64$, $p=0.06)$, although this difference was not statistically significant $(p<0.05)$. By contrast, mean scores on cultural competence did not differ significantly by marital status $(F=0.12, p=.73)$. In view of the fact that there was no variation in either education level or employment status for participants in this study, analysis was not performed on these variables. Based on these preliminary findings indicating little to no differences in the mean scores for implementation of APTA guidelines and cultural competency by demographic characteristics, none of these demographic characteristics were included as covariates in the main study analyses.

Results from the simple linear regression examining the relationship between the implementation of APTA goals and guidelines and the cultural competence of the physical therapy directors indicated that these two variables were not statistically significantly related $(\beta=.24, t=1.65, p=.11)$; therefore the null hypothesis stating that there would be no statistically significant relationship between cultural competence and the implementation of APTA goals and guidelines was not rejected. The overall model fit was not statistically significant $(F=2.71, \quad p=.11)$. Although the relationship between cultural competence and the implementation of APTA goals and guidelines was not statistically significant, it was observed that the variables moved in a positive direction, indicating that as cultural competence increases, so does implemented APTA goals and guidelines. However, given that this relationship was not statistically significant, it may be that these findings do not indicate a true relationship, but are rather due to chance. Based on the findings from the post hoc, it was evident that the observed statistical power based on the sample size and effect size for the linear regression was low.

The mean score for the implementation of APTA goals and guidelines for White/Caucasian participants was 42.40 $(S D=4.25)$, while the mean score for participants who identified as another race/ethnicity was $40.50(S D=1.76)$. The difference between these mean scores was not statistically significant $(t=1.07, p=.29)$; therefore the null hypothesis stating there would be no statistically significant difference in the implementation of these goals and guidelines based on race/ethnicity was not rejected.

The mean cultural competence score for White/Caucasian participants was $4.11(S D=0.51)$, while the mean score for participants who identified as another race/ethnicity was 3.90 $(S D=0.38)$. Results from the independent samples $t$-test indicated that the difference between these mean scores was not statistically significant $(t=0.98, p=.33)$; therefore the null hypothesis stating there would be no statistically significant difference in self-reported cultural competence based on race/ethnicity was not rejected. Although White/Caucasian participants scored higher on the measures of implementation of APTA goals and guidelines and self-reported cultural competence than participants of other races/ethnicities, it is possible that these differences in mean scores were due to 
chance and not a representative of the actual differences between White and non-White physical therapy directors.

\section{Discussion}

While some research has previously been done on the need for diversity and cultural competence in healthcare [7-12, 30, $31]$, diversity and cultural competence in the field of physical therapy [34-36, 39], and to address specific cultural competence training and education [16-18, 21, 25, 40-42], to the best of our knowledge, this study was the first attempt to establish whether the APTA goals and guidelines are being implemented at the director level and whether their implementation is associated with increased cultural competence among physical therapy directors training physical therapists. Findings from these analyses suggest that selfreported cultural competence scores were not significantly related to the implementation of the APTA goals and guidelines within this sample of participating physical therapy directors. Also from the findings, among participating physical therapy directors, race/ethnicity (operationalized as White/Caucasian vs. non-White) was not significantly associated with the implementation of APTA goals and guidelines or with self-reported cultural competence.

There are at least two possibilities for explanation of the findings in this study. First, it might be that the research was underpowered due to low sample size. The post hoc power analysis determined that the power was very low for this regression, suggesting that the sample size obtained via the online survey dissemination may have contributed to the insignificant results. Previous research on response rates for web-based surveys discovered that response rates are typically $11 \%$ lower when surveys are disseminated online than when other dissemination methods are used [43]. In the present study, the survey was distributed to 225 physical therapy directors, and the response rate was $21.3 \%$, which is in line with other studies that relied on web-based surveys [44]. Second, it may be that cultural competency is not necessarily advanced by goals and guidelines, like those implemented by the APTA. It may be useful to consider other theories that could be used to improve cultural competency among physical therapists.

The literature and findings of this study reinforce the theory of culture care of Leininger $[19,45,46]$, who posited that if a healthcare provider and a patient are not culturally suitable for one another, then this can negatively affect the patient's recovery, health, and well-being, but in contrast, if the healthcare provider and the patient are culturally congruent, then the patient can receive care that is beneficial, meaningful and satisfying [47]. It follows that for cultural congruency to persist between therapist and client, the therapist must develop and sustain cultural competence [19, 20]. This process is necessary to enhance the delivery of culturally competent healthcare $[19,20]$. This approach considers the numerous factors that can influence health and healthcare, including acculturation, culture, economy, environment, ethics, ethnohistory, family and kinship, gender, linguistic expressions, philosophy, politics, racial biases, religion, social class, social structural dimensions, and technology. For instance, taking into consideration that culture and context are influencing factors in every phase, from diagnostics to treatment to follow-up care [48], physical therapists would be able to adapt healthcare planning and procedures to take into account the cultural, linguistic, religious, and socioeconomic backgrounds of clients. Physical therapists could be better informed in such a way that when dealing with acculturated clients, they will take into consideration that not all clients retain their traditional culture's perspectives.

Cultural competence programs can inculcate students of physical therapy in cultural competency-related knowledge, attitude, skill, and behavior as each pertains to the culture, gender, race, racial biases, and other perspectives of future clients [49]. This approach would be combined with and/or based on the tested assumptions about how three modalities achieve cultural congruency, (a) culture care preservation and maintenance, (b) culture care adjustment and negotiation, and (c) culture care re-patterning and restructuring [19, 45, 46], and by physical therapists acquiring and developing knowledge to guide the care and treatment of clients: both emic knowledge (detailed local knowledge obtained from within a cultural group, as determined by individual customs, values, and beliefs) and etic knowledge (scientific knowledge acquired from outside a cultural group, as identified by observers or researchers who focus on generalizations about human behavior, including cultural stereotyping) $[19,50]$.

The findings from this study did not support the hypotheses (a) that cultural competence and the implementation of APTA goals and guidelines would differ significantly by the race/ethnicity of the participating physical therapy directors; (b) that there would be a significant difference in self-reported cultural competence based on race/ethnicity; or (c) that there would be a statistically significant relationship between the implementation of APTA goals and guidelines for the training of culturally competent physical therapists and physical therapy directors' self-reported levels of cultural competence. Future research might pursue larger sample populations to verify that there is no significant connection between physical therapy director implementation of APTA goals and guidelines. Also there will be need for further research to reaffirm the application of Leininger's sunrise model in order to inform the training of culturally competent physical therapists by already culturally competent physical therapy directors [51]. In this latter instance, quantitative studies using practicing physical therapists of diverse cultures, races, ethnicities, and genders would complement studies of perceived cultural competence in the workplace. Case studies on cultural competence in the healthcare environment in general and the physical therapy field, in particular, could be tailored to explore evidence of physical therapy directors' cultural competence as it is perceived in the present study. Future research may also compare what physical therapy directors see is being done with what practicing physical therapists think is being done or what is being done in practice. 


\section{Conclusions}

APTA guidelines and goals suggest that cultural competence training is an attainable outcome, and thus that physical therapy can involve the consumer-centricity, equality of access, and client advocacy that the diversity of the healthcare field demands [24, 52]. The present study found no significant link between (a) cultural competence and the implementation of APTA goals and guidelines according to the ethnicity of physical therapy directors, (b) self-reported cultural competence and race/ethnicity, or (c) the implementation of APTA goals and guidelines for the training of culturally competent physical therapists and physical therapy directors' self-reported levels of cultural competence. Hence, cultural competence might be achieved without strict adherence to APTA's goals and guidelines alone [24, 52]. Adequate training for the fundamental alertness of the numerous components that influence health and healthcare as they inform the practice of culturally responsive physical therapy is of the utmost importance. Future recommendations for practicing cultural competency in the field of physical therapy should be supported by further research into larger sample populations to verify that there is no significant connection between physical therapy director implementation of APTA goals and guidelines. Lastly, future research could include the application of Leininger's sunrise model to inform the training of culturally competent physical therapists by already culturally competent physical therapy directors.

\section{Conflicts of Interest}

The authors declare that they have no competing interests.

\section{References}

[1] Colby, S. L., \& Jennifer, M. O. (2017). Projections of the size and composition of the US population: 2014 to 2060: Population estimates and projections. 2017.

[2] Humes, K. R., Jones, N. A., \& Ramirez, R. R. (2010). Overview of race and Hispanic origin: Washington, DC: U.S. Census Bureau. 2011. Retrieved from https://www.census.gov/prod/cen2010/briefs/c2010br-02.pdf.

[3] Heisler, E., \& Shrestha, L. The changing demographic profile of the United States. Congressional Research Service, 7, 5700.

[4] Laveist, T. A., \& Pierre, G. (2014). Integrating the 3DsSocial Determinants, Health Disparities, and Health-Care Workforce Diversity. Public Health Reports. 129 (1_supp12), 9-14. doi: 10.1177/00333549141291s204.

[5] Leavitt, R. (2002). Developing cultural competence in a multicultural world: Part 1. PT Mag Phys Ther, 10 (12), 1-22.

[6] MacMurdie, I. (2017). RC 11-17: Diversity in Physical Therapy? Retrieved April 4, 2019, from https://ptthinktank.com/2017/06/12/rc-11-17-diversity-inphysical-therapy/.
[7] Jeudin, P., Liveright, E., Carmen M. G. D., \& Perkins, R. B. (2014). Race, Ethnicity, and Income Factors Impacting Human Papillomavirus Vaccination rates. Clinical $\begin{array}{llll}\text { Therapeutics. } & 36 & (1), & 24-37 .\end{array}$ 10.1016/j.clinthera.2013.11.001.

[8] Leung, T. F., Ko, F. W., Sy, H. Y., Tsui, S. K., \& Wong, G. W. (2014). Differences in asthma genetics between Chinese and other populations. Journal of Allergy and Clinical Immunology, 133 (1), 42-48. doi: 10.1016/j.jaci.2013.09.018.

[9] Patel, M. I., Schupp, C. W., Gomez, S. L., Chang, E. T., \& Wakelee, H. A. (2013). How Do Social Factors Explain Outcomes in Non-Small-Cell Lung Cancer Among Hispanics in California? Explaining the Hispanic Paradox. Journal of Clinical Oncology, 31 (28), 3572-3578. doi: 10.1200/jco.2012.48.6217.

[10] Romero, C. X., Romero, T. E., Shlay, J. C., Ogden, L. G., \& Dabelea, D. (2012). Changing Trends in the Prevalence and Disparities of Obesity and Other Cardiovascular Disease Risk Factors in Three Racial/Ethnic Groups of USA Adults. Advances in Preventive Medicine, 2012, 1-8. doi: $10.1155 / 2012 / 172423$.

[11] Vaughan, A. S., Rosenberg, E., Shouse, R. L., \& Sullivan, P. S. (2014). Connecting Race and Place: A County-Level Analysis of White, Black, and Hispanic HIV Prevalence, Poverty, and Level of Urbanization. American Journal of Public Health, 104 (7), doi: 10.2105/ajph.2014.301997.

[12] Brusin, J. H. (2012). How cultural competency can help reduce health disparities. Radiologic technology, 84 (2), 129152 .

[13] Adepoju, O. E., Preston, M. A., Gonzales, G. (2015). Health Care Disparities in the Post-Affordable Care Act Era. American Journal of Public Health, 105 (S5). doi: 10.2105/ajph.2015.302611.

[14] Campinha-Bacote, J. (2002). The Process of Cultural Competence in the Delivery of Healthcare Services: A Model of Care. Journal of Transcultural Nursing, 13 (3), 181-184. doi: $10.1177 / 10459602013003003$.

[15] Leininger, M. (1997). Overview of the Theory of Culture Care with the Ethnonursing Research Method. Journal of Transcultural Nursing, 8 (2), 32-52. doi: $10.1177 / 104365969700800205$.

[16] Hayward, L. M., Li, L. (2014). Promoting and Assessing Cultural Competence, Professional Identity, and Advocacy in Doctor of Physical Therapy (DPT) Degree Students Within a Community of Practice. Journal of Physical Therapy Education, 28 (1), 23-36. doi: 10.1097/00001416-20141000000005 .

[17] Matteliano, M. A., \& Stone, J. H. (2014). Cultural competence education in university rehabilitation programs. Journal of Cultural Diversity, 21 (3), 112-118.

[18] Nixon-Cave, K., \& Meadows, J. (2014). Developing Cultural Competence in DPT Students: Is There a Best Practice Educational Model to Facilitate Students Achieving Cultural Competence? Journal of Physical Therapy Education, 28 (1), 37-38. doi: 10.1097/00001416-201410000-00006.

[19] Leininger, M. M. (1991). Cultural Care Diversity and Universality: a Theory of Nursing. New York: National League for Nursing Press. 
[20] Saha, S., Beach, M. C., \& Cooper, L. A. (2008). Patient Centeredness, Cultural Competence and Healthcare Quality. Journal of the National Medical Association, 100 (11), 12751285. doi: 10.1016/s0027-9684(15)31505-4.

[21] Celik, H., Abma, T. A., Klinge, I., \& Widdershoven, G. A. (2012). Process evaluation of a diversity training program: The value of a mixed method strategy. Evaluation and Program Planning, $35 \quad$ (1), 54-65. doi: 10.1016/j.evalprogplan.2011.07.001.

[22] Ansuya, A. (2012). Transcultural nursing: cultural competence in nurses. International Journal of Nursing Education, 4 (1), 5-7.

[23] Truong, M., Paradies, Y., \& Priest, N. (2014). Interventions to improve cultural competency in healthcare: a systematic review of reviews. BMC Health Services Research, 14 (1). doi: 10.1186/1472-6963-14-99.

[24] American Physical Therapy Association. Blueprint for teaching cultural competence in physical therapy education. $2014 . \quad$ Retrieved from https://www.apta.org/Educators/Curriculum/APTA/CulturalCo mpetence/Association of American Medical Colleges. (2016). Underrepresented in medicine definition. Retrieved from https://www.aamc.org/initiatives/urm/.

[25] Thorpe, Q., \& Williams-YorK, B. (2012). Incorporating Cultural Competency into a Physical Therapist Assistant Curriculum. Journal of Best Practices in Health Professions Diversity: Education, Research \& Policy, 5 (2).

[26] Portalla, T., \& Chen, G. M. (2010). The development and validation of the intercultural effectiveness scale. International Communication Studies, 19, 21-36.

[27] Given, L. M. (2008). The Sage Encyclopedia of Qualitative Research Methods. Los Angeles, CA: Sage.

[28] Rossman, G. B., \& Rallis, S. F. (2016). Learning in the Field: An Introduction to Qualitative Research. Los Angeles: SAGE.

[29] Liu, X. S. (2014). Statistical Power Analysis for the Social and Behavioral Sciences: Basic and Advanced Techniques. New York: Routledge.

[30] Budó, M. D. L. D., Schimith, M. D., Alves, C. N., Wilhelm, L. A., Ressel, L. B. (2016). Care and culture: an interface in the nursing knowledge production. Revista de Pesquisa: Cuidado é Fundamental Online, 8 (1), 3691. doi: 10.9789/21755361.2016.v8i1.3691-3704.

[31] Yeowell, G. (2013). 'Isn't it all Whites?' Ethnic diversity and the physiotherapy profession. Physiotherapy, 99 (4), 341-346. doi: 10.1016/j.physio.2013.01.004.

[32] Chaudry, A., \& Beasley, E. (2012). Linguistically competent care for patients with limited English proficiency: A necessity for best practice in physical therapy. HPA Resource, 12 (3), 24-26.

[33] Cleaver, S. R., Carvajal, J. K., Sheppard, P. S. (2016). Cultural Humility: A Way of Thinking to Inform Practice Globally. Physiotherapy Canada, 68 (1), 1-2. doi: 10.3138/ptc.68.1.gee.

[34] Fougner, M., Horntvedt, A. T. (2011). Perceptions of Norwegian physiotherapy students: Cultural diversity in practice. Physiotherapy Theory and Practice, 28 (1), 18-25. doi: 10.3109/09593985.2011.560238.
[35] Grzelak, C. R., Glickman, L. (2014). Reflections on an International Immersion Experience: A Doctor of Physical Therapy Student's Perspective. Journal of Physical Therapy Education, 28 (1), 16-22. doi: 10.1097/00001416-20141000000004 .

[36] Nuciforo, M. A. (2014). Minority Applicants to Physical Therapist Education Programs 2010-2012. Physical Therapy, 95 (1), 39-50. doi: 10.2522/ptj.20130585.

[37] Peiying, N., Goddard, T., Gribble, N., \& Pickard, C. (2012). International Placements Increase the Cultural Sensitivity and Competency of Professional Health Students: A Quantitative and Qualitative Study. Journal of Physical Therapy Education, 26 (1), 61-68. doi: 10.1097/00001416-201210000-00011.

[38] Wehbe-Alamah, H., \& Fry, D. (2014). Creating a Culturally Sensitive and Welcoming Academic Environment for Diverse Health Care Students: A Model Exemplified With Muslim Physical Therapist Students. Journal of Physical Therapy Education, 28 (1), 5-15. doi: 10.1097/00001416-20141000000003 .

[39] Steed, R. (2014). Caucasian allied health students' attitudes towards African-Americans: Implications for instruction and research. The ABNF Journal, Summer, 80-85.

[40] Awosogba, T., Betancourt, J. R., Conyers, \& F. G., et al. (2013). Prioritizing health disparities in medical education to improve care. Annals of the New York Academy of Sciences, 1287 (1), 17-30. doi: 10.1111/nyas.12117.

[41] Oelke, N. D., Thurston, W. E., \& Arthur, N. (2013). Intersections between interprofessional practice, cultural competency and primary healthcare. Journal of Interprofessional Care, 27 (5), 367-372. doi: $10.3109 / 13561820.2013 .785502$.

[42] Renzaho, A. M. N., Romios, P., Crock, C., \& Sonderlund, A. L. (2013). The effectiveness of cultural competence programs in ethnic minority patient-centered health care--a systematic review of the literature. International Journal for Quality in Health Care, 25 (3), 261-269. doi: 10.1093/intqhe/mzt006.

[43] Manfreda, K. L., Bosnjak, M., Berzelak, J., Haas, I., \& Vehovar, V. (2008). Web Surveys versus other Survey Modes: A Meta-Analysis Comparing Response Rates. International Journal of Market Research, 50 (1), 79-104. doi: $10.1177 / 147078530805000107$.

[44] Biersdorff, K. K. (2009). How many is enough? The quest for an acceptable survey response rate. Bright Ideas. https://kkbiersdorff.wordpress.com/2009/09/16/how-many-isenough/. Published September 16, 2009. Accessed April 20, 2019.

[45] Leininger, M. (1992). Theory of culture care and uses in clinical and community contexts. In M. Parker (Ed.), Theories on nursing (pp. 345-372). New York, NY: National League for Nursing Press; 1992.

[46] Leininger, M. (1995). Transcultural nursing (2nd ed.) New York, NY: McGraw-Hill; 1995.

[47] Zoucha, R., Husted, G. (2000). The Ethical Dimensions Of Delivering Culturally Congruent Nursing And Health Care. Issues in Mental Health Nursing, 21 (3), 325-340. doi: $10.1080 / 016128400248121$. 
[48] Synnott, A., O'Keeffe, M., Bunzli, S., Dankaerts, W., Osullivan, P., \& Osullivan, K. (2015). Physiotherapists may stigmatise or feel unprepared to treat people with low back pain and psychosocial factors that influence recovery: a systematic review. Journal of Physiotherapy, 61 (2), 68-76. doi: 10.1016/j.jphys.2015.02.016.

[49] Doherty, D., Maher, S. F., Ivanikiw, C., Hales, M., Lebiecki, T., \& Wren, P. A. (2017). Perceptions of cultural competency in doctor of physical therapy students introduction. Journal of Cultural Diversity, 24 (2). Retrieved from EBSCOhost.

[50] Kottak, C. P. (2018). Mirror for Humanity: a Concise Introduction to Cultural Anthropology. New York, NY: McGraw-Hill Education.
[51] Chu, J., Leino, A., Pflum, S., \& Sue, S. (2016). Psychotherapy With Racial/Ethnic Minority Groups: Theory and Practice. In L. E. Beutler, A. J. Consoli, \& B. Bongar (Eds.), Comprehensive textbook of psychotherapy: Theory and practice (pp. 346-362). Oxford University Press; 2016.

[52] American Physical Therapy Association. (2012). Operational plan on cultural competence. Retrieved from http://www.apta.org/uploadedFiles/APTAorg/About Us/Polici es/APTA_Adopted_Plans/OperationalPlanCulturalCompetenc e.pdf\#search $=\% 22$ Facilitatethedevelopmentofculturallycompe tentphysicaltherapists\%22APTA_Adopted_Plans/Operational PlanCulturalCompetence.pdf. 